\title{
LAS INCUBADORAS UNIVERSITARIAS DE ECONOMÍA SOLIDARIA EN BRASIL - UN ESTUDIO DE CASOS
}

\author{
POR \\ Fátima CRUZ-SOUZA ${ }^{2}$, \\ Ana Lucia CORTEGOSO, \\ Maria ZANIN e \\ Ioshiaqui SHIMBO 3
}

\section{RESUMEN}

La Economía Solidaria es un término que designa un movimiento social que viene creciendo en Brasil durante los últimos 15 años e incluye un gran conjunto de actores socioeconómicos. En él se generan alternativas de inserción productiva para colectivos socialmente desfavorecidos, pero sus objetivos no son solamente los cambios económicos, sino la promoción de cambios socioculturales hacia una sociedad más igualitaria y solidaria.

Para apoyar y fortalecer la economía solidaria se crearon desde algunas universidades brasileñas las Incubadoras de Cooperativas Populares. En el presente estudio se analiza la trayectoria de la Incubadora Regional de Cooperativas Populares de la Universidad Federal de São Carlos, situada en el Estado de São Paulo (Brasil), como estructura que conjuga la investigación, la docencia y la extensión universitaria dirigidas hacia los procesos de incubación de emprendimientos solidarios. Aplicando el estudio de casos se sistematizan los avances conceptuales y metodológicos que se relacionan con las dificultades encontradas por

\footnotetext{
${ }^{1}$ Las autoras y autores agradecen a las siguientes entidades financiadoras: FAPESP, CNPQ, PRONINCMDS/MEC, PRONINC- FINEP, Ayuntamiento de São Carlos, Universidade Federal de São Carlos, y a todas las entidades que vienen colaborando con el proyecto.

${ }^{2}$ Universidad de Valladolid (España). Dirección de correo electrónico: fcruz@psi.uva.es

3 Universidade Federal de São Carlos (Brasil). Direcciones de correo electrónico: cortego@ufscar.br; dmza@power.ufscar.br; shimbo@power.ufscar.br, respectivamente.

REVESCO N 106 - Tercer Cuatrimestre 2011 - ISSN: 1885-8031 - www.ucm.es/info/revesco
}

DOI: 10.5209/rev_REVE.2011.v106.37373

Fecha de recepción: 30/06/2010

Fecha de aceptación: 24/10/2010 
estos emprendimientos para su consolidación y la creación de redes de iniciativas económicas solidarias en el territorio estudiado.

El examen de este proceso ayudó a identificar y discutir estrategias para el progreso de los argumentos conceptuales, de la metodología de asesoramiento y para la aplicación a otras iniciativas similares.

Palabras clave: Economía solidaria, desarrollo territorial, incubadoras universitarias, método de incubación.

\title{
THE UNIVERSITY INCUBATORS OF SOLIDARITY ECONOMY IN BRAZIL - A CASE STUDY
}

\begin{abstract}
The Solidarity Economy is a social movement who is growing up in the last 15 years in Brazil and it includes a very big group of socioeconomic actors. This movement improves alternatives of productive insertion to disadvantage groups of population. However its objectives are not only the economic changes, but the promotion of social and cultural changes toward a more equal and solidarity society.
\end{abstract}

To support and strengthen the solidarity economy, the Popular Cooperatives Incubators had been created in some Brazilian Universities. In this study we analyse the path of the Regional Incubators of Popular Cooperatives of Federal University of San Carlos (State of São Paulo, Brazil). It works as a structure which combines research, learning and university extension to improve incubation process of solidarity entrepreneurship. Through the case study we try to know the conceptual and methodologial progress that is related with the difficulties founded by the consolidation of solidarity entrepreneurs and to creation of networks between solidarity economics initiatives in their territories. 
The study of this process helps us to identify and discuss about strategies to improve the conceptual framework and the advice methodology, and to transfer the results to other initiatives.

Keywords: Solidarity Economy; territorial development; university incubators; incubation method.

\section{ECONOMÍA SOLIDARIA COMO UNA FORMA ALTERNATIVA DE GENERACIÓN DE TRABAJO, RENTA Y RELACIONES SOCIALES}

En las últimas dos décadas del siglo XX, con la hegemonía neoliberal instituida, ha sido posible notar el agravamiento de las condiciones sociales de una gran parte de la población de los países subdesarrollados. A pesar de que las afirmaciones realizadas por los defensores de esa política, nos muestren que los mercados libres producen mayor eficiencia en el bien-estar de todos, lo que en realidad manifiesta es una concentración de renta y un aumento de la pobreza y de la exclusión social. Problemas como bajo nivel educacional, inaccesibilidad a los servicios de salud, altos índices de desempleo y precarización del trabajo, aumento de la violencia y de la criminalidad, destrucción de la familia, entre muchos otros, producen círculos perversos de exclusión que solo tienden a generar más pobreza, tornando el cuadro de la situación social cada vez más insostenible (KLISBERG, 2002). Los trabajadores, una vez expuestos al desempleo, a la subocupación, al trabajo precarizado, o informal, buscan alternativas que les generen una renta donde al contrario de buscar lucro, son reguladas por objetivos de reproducción ampliada de la vida y de la emancipación económica y social.

La Economía Solidaria surge entonces, como alternativa de generación de trabajo y renta para aquellos que fueron excluidos del mercado capitalista. Sus emprendimientos se dan en las esferas de la producción, de la distribución, del consumo, del ahorro y del crédito y pueden tomar la forma de cooperativas, asociaciones, clubes de trueques, empresas autogestionarias, fondos rotativos, etc. Tales prácticas económicas están creciendo no solamente en Brasil, sino en varios países de Suramérica, así como en varias otras partes del 
mundo (SINGER, 2001; ARROYO, 2006). Según el Fórum Brasileiro de Economía Solidaria (EBES), la economía solidaria es:

“fruto de la organización de trabajadores y trabajadoras en la construcción de nuevas prácticas económicas y sociales fundadas en relaciones de colaboración solidaria, inspiradas por valores culturales que colocan el ser humano como sujeto y finalidad de la actividad económica, en vez de la acumulación privada de la riqueza en general y del capital en particular” (FBES, 2006, p.3).

A lo largo de los últimos quince años, la Economía Solidaria en Brasil viene ganando amplitud como movimiento social y también como objetivo de las políticas públicas de los gobiernos federales, estaduales y municipales. Por esto, es posible notar el aumento de los estudios académicos sobre este tema, así como el surgimiento de nuevas líneas y objetos de las investigaciones que los caracterizan. Incubadoras universitarias de emprendimientos solidarios han surgido en Brasil, desde mediados de la década de 1990, como forma de promover tanto la producción como el acceso al conocimiento por parte de la población más excluida, fomentando la Economía Solidaria como una forma, tanto de generación de renta como de otra manera de establecer relaciones entre personas y de ellas con su ambiente, en atención a sus necesidades en todas las esferas: económica, social, ambiental y psicológica (SINGER 2002a)

\section{INCUBADORAS DE EMPRENDIMIENTOS ECONÓMICOS SOLIDARIOS EN LAS UNIVERSIDADES BRASILEÑAS}

Según el Ministerio del Trabajo y Empleo (MTE, 2010) del gobierno brasilero, "Las primeras incubadoras de cooperativas y de otros emprendimientos económicos solidarios fueron creadas en las universidades brasileñas, como espacios de socialización de los diversos conocimientos producidos en estas instituciones, favoreciendo la articulación entre enseñanza-investigaciónextensión y la integración de las universidades con comunidades y segmentos externos a la comunidad académica. Así, aunque guarden similitudes con las incubadoras universitarias de empresas, la especificidad de las incubadoras de 
cooperativas reside en el trabajo con emprendimientos colectivos y de base autogestionaria, recién creados o en vía de creación, que exigen procesos específicos de formación, asesoría y asistencia técnica, en aspectos administrativos, productivos y organizativos, con el objetivo principal de contribuir para su autonomía y sustentabilidad”. (MTE, 2010)

Actualmente, en más de una centena de universidades e instituciones de enseñanza superior brasileñas están implantadas incubadoras, organizadas en redes para desarrollar conocimiento y procesos de intervención en la temática de Economía Solidaria. La Red Universitaria de Incubadoras Tecnológicas de Cooperativas Populares (Rede de ITCPs) fue iniciada en 1998 y está constituida actualmente por ITCPs de 44 universidades en cinco regiones de Brasil. Tiene como objetivo desarrollar y diseminar conocimientos sobre autogestión y economía solidaria, dentro y fuera de las universidades, además de estimular en la universidad, el desarrollo de otras tecnologías específicas, elaboradas para atender las actividades productivas de los emprendimientos y sus estrategias. Esta red tiene como objetivo vincular de forma interactiva y dinámica las incubadoras universitarias, favoreciendo el intercambio de tecnologías y de conocimientos entre ellas y con la sociedad (ITCP-USP, 2010). Además, la Red Universitaria Unitrabalho, agrega, actualmente, 92 universidades e instituciones de enseñanza superior de Brasil. Fue creada en 1996, con el objetivo de contribuir a saldar la deuda social que las universidades brasileñas tienen con los trabajadores (UNITRABALHO, 2010)

Volver el conocimiento científico y tecnológico accesible a la producción históricamente excluida es un desafío para las universidades públicas, principalmente si esto incluye cuestionamientos a los modos hegemónicos de producción, distribución y consumo de bienes y servicios, que enfatizan la competitividad, el individualismo y la degradación ambiental Además, implica buscar otros modos que privilegien la cooperación, la solidaridad y la autogestión; los cuales son principios fundamentales de la Economía Solidaria. El desafío se hace aún más complejo, al producir conocimiento científico (comprensión de conceptos y fenómenos) y tecnológico (transferencia) paralelamente a la actuación en la realidad social con poblaciones excluidas y con recursos escasos (financieros, económicos, cognitivos etc.), y a la formación de diferentes tipos de personas para cambios de conductas significativas, en la perspectiva de mejorar las condiciones de vida de la mayoría de la población. 
En tal contexto, la Incubadora Regional de Cooperativas Populares de la Universidad Federal de São Carlos (INCOOP/ UFSCar), que forma parte de la Red de ITCPs, viene, desde 1998, implementando actividades de enseñanza, investigación y extensión de forma articulada, relevantes para la Economía Solidaria, con la participación de docentes, profesionales y alumnos de diferentes áreas del conocimiento y campos de actuación profesional. Entre tales actividades se destacan las que se refieren a los procesos de incubación de emprendimientos económicos solidarios (EES) en diferentes sectores económicos.

\section{LA INCUBADORA REGIONAL DE COOPERATIVAS POPULARES DE LA UNIVERSIDAD FEDERAL DE SÃO CARLOS}

En 1998 la Incubadora Regional de Cooperativas Populares de la Universidad Federal de São Carlos - INCOOP/UFSCar, situada en la ciudad de São Carlos, Estado de São Paulo (Brasil) inició sus contactos con la comunidad, para presentar posibilidades de organización del trabajo colectivo. Disponía, en esa época, de apenas algunas referencias teóricas y algunas experiencias, aún iniciales, como orientaciones para su trabajo de "incubar" cooperativas populares, como forma de superación de la exclusión y de construcción de alternativas a un sistema económico y social que viene produciendo muchos más problemas que condiciones dignas de vida en el planeta. En los años de dedicación a la producción y transformación de conocimiento en iniciativas humanas en Economía Solidaria, varios segmentos de la población fueron atendidos, algunos de forma más completa y otros más parcialmente, con una perspectiva de construir emprendimientos solidarios, aunque en algunas ocasiones no llegasen a realizarse. En estos procesos de asesoría se dieron avances y equivocaciones, muchas veces, derivados de intentos de intervenir sin el conocimiento suficiente, de osadía compensada por el esfuerzo de sistematizar las experiencias, evaluarlas empírica y conceptualmente y, por lo menos, hacerlas públicas, como condición de visibilizar lagunas de conocimiento o para ayudar a suplir dichas lagunas. La opción por el compromiso radical con la no separación entre enseñanza, investigación y extensión, y el desafío de lograr eso a través del contacto directo con los aspectos de la realidad que constituyen problemas sociales significativos, a la vez, transformando el conocimiento disponible en conductas humanas, produciendo conocimiento nuevo y, en este proceso, garantizando la formación para la 
Economía Solidaria a todos los niveles, trajo consigo exigencias metodológicas considerables. El desafío, aún mayor, ha sido realizar todo ello de un modo articulado con otras entidades, con diferentes actores sociales, con puntos de interés también diversificados dentro de la propia Economía Solidaria.

Formular preguntas y buscar respuestas sobre la posibilidad de que la Economía Solidaria desempeñe un papel relevante en los cambios que, aún aquellos que no creen en esa posibilidad reconocen que son necesarios para la construcción de un mundo menos desigual,representó y representa la contribución de profesores, alumnos, profesionales de nivel superior y trabajadores implicados en la INCOOP en más de 10 años de su existencia. En su equipo de trabajo, la INCOOP/UFSCar ha contado con la participación de docentes y alumnos de diferentes áreas del conocimiento (Psicología, Ingeniería Civil, de Materiales, de Producción, Letras, Enfermería, Terapia Ocupacional, Ciencias Sociales, Pedagogía, entre otros) y de profesionales de diferentes campos de actuación profesional (Arquitectura, Psicología, Pedagogía, Ciencias Sociales, Biología, Agronomía, Química, entre otros).

La INCOOP, desde el inicio de su funcionamiento, sistemáticamente viene atendiendo y contribuyó directamente para la formación de al menos 20 emprendimientos solidarios, en áreas como alimentación, limpieza y vigilancia, lavandería, reciclaje, costura, producción de plantones, artesanías, carpintería y producción agropecuaria y productos de limpieza. posibilitando así, la creación de trabajo y de renta para aproximadamente 500 personas. La forma de actuación en el atendimiento a la comunidad y para la constitución de emprendimientos solidarios, corresponde al llamado "método de incubación" de la INCOOP, un proceso que será detallado más adelante.

Aunque la incubación de emprendimientos económicos solidarios constituya la centralidad de la actuación de la INCOOP, ésta no es la única actividad realizada por el equipo de trabajo, como forma de atender a su compromiso con la enseñanza, investigación y extensión. En el ámbito de la enseñanza de nivel superior, la Incubadora mantiene desde 2003, una oferta de asignatura semestral destinada a alumnos de cualquier curso de grado interesados en la temática de la Economía Solidaria.Además de la familiarización con los conceptos relevantes en este campo, los participantes de dicha asignatura se insertan en los proyectos de intervención que en ese momento se estén desarrollando en la Incubadora, 
iniciando su aprendizaje en relación a la producción de conocimiento por medio de formulación de preguntas de investigación y participando de actividades que profundizen determinadas temáticas específicas. Todo ello se da en un contexto en que la multidisciplinariedad se materializa por medio de un equipo de trabajo realmente multidisciplinar, y por la presencia de alumnos en proceso de formación provenientes de diferentes especialidades. En la esfera de la enseñanza de posgrado, desde 2009, se imparte la disciplina de Economía Solidaria, Ciencia y Tecnología para el alumnado del Programa Multidisciplinar en Ciencia, Tecnología y Sociedad de la UFSCar. Además, la INCOOP constituye un campo para prácticas curriculares y ofrece condiciones para el desarrollo de trabajos de investigación con respecto a los grados, para diferentes cursos y posgrados, a partir de diferentes programas que cuentan con la participación de los profesores trabajote la INCOOP. Diversos alumnos y alumnas de posgrado desarrollan sus propios proyectos simultáneamente a actividades de intervención en proyectos de la Incubadora.

La INCOOP actúa, aún, en el fomento de iniciativas de apoyo a los emprendimientos incubados, que responden a la promoción de finanzas solidarias y de prácticas de consumo ético, responsable y solidario; en la realización y divulgación de investigaciones realizadas según las líneas en que trabaja cada docente del equipo de trabajo, en el ámbito del grupo de investigación en que están vinculados los miembros del grupo (Economía Solidaria y Cooperativismo Popular), incluyendo sus propias prácticas de asesoría, orientadas por el siguiente conjunto de directrices: a) público prioritario de procesos de producción de conocimiento: fenómenos y procesos incluidos, o de interés para el público a quien se dirige la INCOOP (excluidos: desempleados, trabajadores precarizados, moradores de barrios pobres, personas con necesidades especiales, poblaciones en desventaja social, en situaciones de riesgo); b) Rigor ético compatible, en el grado máximo posible, con exigencias establecidas por normas y con la lógica de la Economía Solidaria, que prevé protagonismo por parte de la poblaciones implicadas, y empoderamiento de estas poblacionespoblaciones por medio, también, del proceso de producción de conocimiento; c) Relevancia: del punto de vista social (beneficios previstos, ¿quiénes son los beneficiarios directos e indirectos, en corto, medio y largo plazo?); del punto de vista científico (¿a qué laguna del conocimiento corresponde? ¿Qué contribución ofrece para los avances del conocimiento?); histórico (justificativa para la implementación de proyectos en el contexto histórico); d) inclusión de mecanismos para acceder al conocimiento producido para quien lo necesita, como parte del 
proceso de producción del conocimiento; e) Explicitación de mecanismos para el empoderamiento (CRUZ et al. (2006) del público al que está dirigida la INCOOP durante la implementación de los procesos de producción de conocimiento y a partir de sus resultados.

La Incubadora participa también, como agencia de fomento, de actividades directamente relacionadas al llamado movimiento de la Economía Solidariaa nivel del municipio donde se ubica la universidad, de la región o estado en que está localizada, en el ámbito nacional, y en el ámbito de los foros de Economía Solidaria. Conjuntamente con otros actores del movimiento, actúa de modo a alcanzar una estructura legal apropiada a la lógica de la Economía Solidaria y en la implantación de políticas públicas de Estado, como fomento a esta forma de producción y organización social. En la ciudad en que actúa, la INCOOP contribuyó para la constitución de un Centro Público de Economía Solidaria, para la formulación de una Ley Local de Economía Solidaria y su aprobación, y para la constitución del Foro Municipal de Economía Solidaria. Participa y actúa, así mismo, en el fomento y estudio de iniciativas de articulación del movimiento de la Economía Solidaria, tales como la Red de Incubadoras Tecnológicas de Cooperativas Populares (con más de 40 incubadoras distribuidas por Brasil), y en instancias internacionales, como la Asociación de Universidades Grupo Montevideo (AUGM), como representante de la UFSCar en el Comité para Asuntos Cooperativos y Asociativos (PROCOAS), y viene ampliando sus alianzas internacionales en España (Universidad de Valladolid) e Italia (Cooperativas sociales), entre otras.

\subsection{La INCOOP y su método de incubación.}

El examen y la sistematización de las experiencias de incubación llevadas a cabo por la INCOOP han sido formas privilegiadas de producción del conocimiento, tanto para apoyar el propio trabajo de la Incubadora, como para la continuidad de estudios y aplicaciones en el ámbito de la Economía Solidaria. En este sentido, durante estos años de actuación vienen construyéndose un referencial de abordaje, a partir de las varias experiencias de incubación, que está constituido por un conjunto de comportamientos de referencia de la Incubadora, denominado método de incubación. Hay que señalar, sin embargo, que éstese encuentra en permanente proceso de revisión. 
En el ámbito de la INCOOP, se entiende por incubar emprendimientos solidarios, el hecho de asesorar grupos para la formación de emprendimientos económicos solidarios, por medio de la oferta de apoyo técnico y de acompañamiento del proceso de toma de decisiones e implementación de actividades. Las personas responsables por la incubación participan en todas las etapas del trabajo, incluyendo la evaluación de resultados, por medio de relaciones dialógicas, siempre que se presenten demandas u oportunidades que sean compatibles con los criterios en vigor dentro de la INCOOP, y considerando la disponibilidad de recursos necesarios. Como resultado de este proceso se intenta alcanzar la existencia y consolidación de emprendimientos solidarios y autogestionarios de naturaleza popular, organizados para el trabajo colectivo, que funcionen de forma autónoma, con capacidad para identificar sus propias necesidades y, así mismo, buscar las alternativas para que sean atendidas, incluidas en el mercado y en el contexto más amplio de la Economía Solidaria y con características gradualmente más compatibles con principios de Economía Solidaria.

Tomando como punto de partida esta caracterización general del proceso de incubar emprendimientos solidarios, a partir de la experiencia en este campo y de reflexiones conceptuales, fue identificado un conjunto de comportamientos más específicos y relevantes para alcanzar los resultados previstos para asesorar los grupos atendidos. Presentamos a continuación una indicación sintética de estos comportamientos sistematizada por CORTEGOSO et al. (2005 y 2008):

1) Procesar demandas para incubación de emprendimientos solidarios, recibidas de diferentes actores sociales, de modo que éstas sean aceptadas o rechazadas en función de criterios establecidos por el equipo de trabajo de la INCOOP y de recursos existentes para la incubación de nuevos emprendimientos, de forma transparente para todos los implicados y con orientaciones realizadas en aquellos casos en que la demanda no puede ser atendida;

2) Identificar poblaciones con potencialidades de formación de emprendimientos solidarios, compatibles con la demanda, cuando esta población no está ya identificada en la demanda;

3) Caracterizar cada uno de los actores, de diferentes tipos, implicados o que deban ser incluidos en el proceso de incubación especifico a ser realizado, de forma más completa posible, como condición para elaborar propuestas de actuación junto al 
grupo y, de preferencia, con participación efectiva de los ya implicados, particularmente la población potencial para la formación del grupo de trabajo productivo;

4) Presentar la Economía Solidaria como posibilidad de organización enfocando la generación de trabajo y renta a la población o grupo potencial para la constitución de emprendimientos solidarios.De modo que cada uno de los miembros de dicha población sea esclarecido con respecto a las características de los emprendimientos en el ámbito de la economía solidaria, principios del cooperativismo, alternativas existentes para la generación de trabajo y renta, forma y condiciones de trabajo de la INCOOP y sea competente en el uso y comprensión del lenguaje y de los conceptos en el campo de la Economía Solidaria, que pueda escoger de forma libre y esclarecida sobre la adhesión a la propuesta cooperativista y contribuir a la búsqueda de recursos para viabilizar el atendimiento de la INCOOP, en caso de que fuera necesario;

5) Apoyar el grupo en su organización inicial para que los participantes puedan tomar decisión democrática y esclarecida sobre la formación (o no) del emprendimiento económico solidario, en caso tal, que el grupo presente un grado insuficiente de organización autónoma para eso;

6) Elaborar una propuesta conjunta de trabajo, con la participación de los miembros con potencialidades dentro del grupo a ser "incubado", que indique, lo más claro posible los acuerdos colectivos sobre responsabilidades mutuas en el proceso de incubación, objetivos, metas, plazos, formas de trabajo, contrapartidas y otros aspectos que puedan contribuir para la regulación de las conductas de todos los y las participantes, y que al mismo tiempo, sirvan como parámetros para evaluar el trabajo, atendiendo las necesidades, intereses y posibilidades de todas las personas implicadas; 7) Apoyar la selección de la actividad económica a ser realizada por el grupo a partir del examen de cadenas productivas, de modo que ésta presente viabilidad económica, relevancia social y compatibilidad con los recursos materiales y con el capital humano disponible, con recursos adicionales e infraestructura necesaria para la implementación de la actividad, éstos, debidamente identificados y potencialmente accesibles;

8) Promover la formación de los miembros del grupo sobre el tema del cooperativismo popular y la Economía Solidaria de forma continua y permanente, aumentando la autonomía del grupo y su inserción en el contexto general de la 
Economía Solidaria, así como, aumentando las probabilidades de que los miembros de los emprendimientos atendidos presenten conductas gradualmente más compatibles con la cultura de la Economía Solidaria;

9) Promover la formación continua y permanente de los miembros, sobre la autogestión y la gestión administrativa, de modo que todos los miembros del emprendimiento económico solidario tengan oportunidades de aprendizaje, siendo gradualmente más capaces de participar de su administración, utilizando técnicas y procedimientos adecuados y compatibles con los principios del movimiento de la Economía Solidaria; trabajadores y trabajadoras con control sobre todo el proceso económico (producción, financiamiento, administración, distribución, trueque y consumo ético, crítico, consciente de los frutos de su trabajo);

10) Promover condiciones para la capacitación técnica de los participantes en relación al servicio o producto ofertado por el emprendimiento, de forma permanente, de modo que sea posible mantener y ampliar la viabilidad económica del emprendimiento, generando mejores condiciones para reacceder a los mejores puestos y condiciones de trabajo. Que el grupo esté preparado para mantenerse buscando oportunidades de capacitación técnica de forma autónoma y para establecer alianzas en este sentido, de modo que cada miembro pueda alcanzar un desempeño técnico de alta calidad, mejoría de la autoestima y mejores condiciones para colaborar en el grupo;

11) Promover la elaboración de normas de funcionamiento del emprendimiento, particularmente el reglamento interno, de manera participativa, lo más temprano posible y de modo que éstas indiquen claramente comportamientos esperados de los miembros y del emprendimiento, definidos por el grupo de acuerdo con sus necesidades y características, de forma compatible con la legislación existente y de manera a minimizar la probabilidad de conflictos y distorsiones en relación a los principios de la economía solidaria. Así mismo, debe constar claramentelas consecuencias que sean capaces de mantener dichos comportamientos, por lo que agregan o garantizan efectos benéficos para cada individuo y para el grupo en términos de satisfacción de sus necesidades y alcance de sus objetivos, además, con especificación de las consecuencias del no cumplimiento de las reglas, que sean viables y proporcionales a la gravedad de las conductas que generan desvíos, de modo a promover la compresión de los miembros sobre la función de cada norma. 
12) Apoyar el grupo en la legalización del emprendimiento, de modo que éste quede regularizado de acuerdo con la definición del grupo y su naturaleza jurídica en las leyes vigentes, con el menor coste posible, y con miembros lo más capacitados que sea posible en relación a este proceso;

13) Apoyar al grupo en la implantación del emprendimiento, de modo que éste tenga su inserción inicial en el mercado, con capacidad de funcionar gradote la mejor manera posible, de acuerdo con los principios cooperativistas y de la Economía Solidaria, con normas internas mínimas establecidas y con capacidad de autogestión y gestión administrativa, posibilitando evaluaciones del funcionamiento y ampliación de las condiciones de su viabilidad económica.

14) Apoyar al grupo a implementar un sistema de control por medio de indicadores sobre su propio financiamiento, de modo que pueda ser utilizado de forma habitual con el grupo, produciendo de forma autónoma datos sobre su propio funcionamiento, de modo a subsidiar sus decisiones tácticas, estratégicas y cotidianas; además, el sistema a implantar debe seguir un alto grado de compatibilidad con los otros sistemas de control dentro de la Economía Solidaria;

15) Apoyar el grupo en la implementación del emprendimiento, de forma eventual y esporádica, de modo que se pueda mantener incluido en el mercado, funcionando de forma creciente en armonía con los principios cooperativistas y de la Economía Solidaria, contando con aliados y articulándose con otros emprendimientos solidarios, particularmente los de la cadena productiva de la cual forme parte, con un creciente grado de autonomía en relación a la Incubadora, con derecho o dominio creciente de los medios de producción,;

16) Apoyar el grupo para que participe en redes de cooperación y en iniciativas del movimiento de Economía Solidaria, de modo que el emprendimiento esté articulado con otros emprendimientos solidarios, apoyado por otros emprendimientos del campo de la economía solidaria, participando del mayor número posible de iniciativas organizativas de este campo, tanto para la viabilidad de la actividad productiva como para la defensa política de este campo.

Aun siendo estos comportamientos considerados relevantes, no siempre todos son necesarios en todas las situaciones. Por ejemplo, la identificación de personas con potencial para la participación dentro del emprendimiento, no se justifica cuando estas personas ya 
están debidamente identificadas desde el inicio del proceso. De la misma manera, la lista citada anteriormente no indica una secuencia única de desarrollo de las actividades comprendidas en el método de incubación, siendo muy frecuente que varias ocurran simultáneamente. En cada situación, es necesario examinar las condiciones específicas relacionadas con la demanda o la oportunidad de incubar un emprendimiento, y promover las adaptaciones deseables para un buen atendimiento de estas peculiaridades, sin perder de vista los aspectos esenciales del proceso de incubación, en la dirección de los resultados de la Incubadora, enunciados como una actuación deseable.

\subsection{La INCOOP y su estrategia de desarrollo territorial}

La experiencia de la INCOOP/UFSCar y un profundo debate llevaron a un cambio de perspectiva en losúltimos años. Constituyéndose la atención a los territorios como forma de actuación preferencial, más que la atención a los emprendimientos aislados. Estos territorios fueron definidos a partir de los propios emprendimientos económicos solidarios que la Incubadora había atendido y que presentan un significativo potencial para la ampliación y consolidación de la Economía Solidaria. Sin embargo, se establece la consolidación de la Economía Solidaria en articulación con otras políticas sectoriales, como salud, educación, vivienda y saneamiento ambiental, como forma de desarrollo territorial, enfatizando la generación de renta por medio del trabajo colectivo y de prácticas de cooperación. Por lo tanto, presuponemos que los principios que caracterizan los emprendimientos económicos solidarios: autogestión, democracia, participación, igualitarismo, cooperación, autosustentación, desarrollo humano y responsabilidad social (GAIGER, 2004), pueden expandirse, transformándose en una estructura para un proceso más amplio y que abarque los sistemas cultural, social y político de una cierta comunidad, localidad, región o territorio.

Para promover el desarrollo local basado en los principios de la Economía Solidaria, la INCOOP/UFSCar buscó recursos en diversas agencias de fomento, por medio de elaboración de proyectos. Desde 2007, la estrategia de actuación de la INCOOP/UFSCar en todos sus proyectos está centrada en la ampliación y articulación de iniciativas de Economía Solidaria para el desarrollo local en un territorio urbano y en un territorio rural específico. Esto viene dándose por medio de la consolidación de los emprendimientos solidarios ya existentes y de su protagonismo en el proceso de creación e implantación de otros emprendimientos e 
iniciativas solidarias en estas áreas. Como resultado, se espera la ampliación, de forma articulada, de iniciativas de Economía Solidaria, incluyendo productores dentro de una misma cadena productiva, prestadores de servicios, consumidores, gestores públicos y otros actores que fomenten la Economía Solidaria, con perspectiva de desarrollo territorial sustentable, seguridad alimenticia y nutricional, y soberanía alimenticia.

Partiendo de las iniciativas económicas de la Economía Solidaria existentes en dos barrios de la ciudad de São Carlos (SP, Brasil), y que además constituyen el territorio urbano de actuación de la INCOOP/UFSCar, así como de necesidades ya identificadas por las personas que componen estos emprendimientos, los miembros del equipo de trabajo de la INCOOP/UFSCar que ya apoyaban tales iniciativas y otros actores sociales que desenvuelven actividades en favor de la mejoría de la calidad de vida de los habitantes, vienen desarrollando un proceso de caracterización de las demandas, identificación de recursos y oportunidades existentes y/o potenciales en el territorio - , y de definición de cadenas productivas a ser promovidas en la región, por medio de la constitución de nuevos emprendimientos solidarios.

En un periodo de seis meses, y contando con recursos obtenidos a través de las agencias de financiación, fue posible establecer convenios con más de 20 entidades asociadas, las cuales también son actores sociales: Ayuntamiento y diferentes organismos municipales, grupos religiosos, emprendimientos solidarios, sectores universitarios y organizaciones sociales con objetivos diversos. Al mismo tiempo, se ha podido identificar propuestas de cadenas productivas como referencia para los estudios de viabilidad e implantación de iniciativas solidarias, en las áreas de limpieza, alimentación, atención a personas dependientes, servicios logísticos, vivero de plantas, producción de pequeños objetos de madera, cultura, entre otros.

\subsection{Breve Caracterización de los territorios enfocados por la INCOOP/UFSCar}

Como se ha señalado anteriormente, la INCOOP/UFSCar, desde el 2007, ha puesto en práctica su estrategia de realizar intervenciones que traten el desarrollo territorial, por medio del fortalecimiento y de la expansión de emprendimientos e iniciativas de la Economía Solidaria en regiones habitadas por poblaciones en riesgo de exclusión social, articulando 
diferentes actores sociales, sensibilizando y capacitando la creación de redes de colaboración. Hasta el momento fueron delimitados dos territorios, uno en área rural y otro en área urbana.

\section{Territorio urbano}

El territorio urbano al que se refieren las actividades de la asesoría por parte de la INCOOP/UFSCar, está afectado por una situación de gran pobreza en un municipio del interior del Estado de São Paulo, el cual, aunque presente alto Indice de Desarrollo Humano (IDH) - considerando las medias nacionales y regional - no está libre del perfil de desigualdad, producido por el crecimiento capitalista, generador de exclusión.

El municipio de São Carlos tiene como ejes dominantes de su economía los sectores industrial y de servicios, además de la producción agropecuaria (leche, naranjas y caña de azúcar). La implantación de dos grandes universidades (USP y UFSCar en las décadas de 50 y 60 respectivamente) aliada al consecuente surgimiento de diversas empresas hizo con que São Carlos ganara la condición de polo tecnológico y fuera conocida como la Capital de la Tecnología. A pesar de esta consideración, la ciudad posee grandes bolsas de pobreza, entre ellas el Jardín Gonzaga y el Jardín Monte Carlo, dos barrios del territorio urbano, centros de los estudios y de la intervención de la INCOOP.

Estos dos barrios, situados en área fronteriza del perímetro urbano de la ciudad de São Carlos, poseen los mayores índices de vulnerabilidad social de la ciudad (extrema pobreza, altos índices de violencia, de desempleo, de drogadicción, de baja escolaridad y de niñez y adolescencia en riesgo personal y social), comenzó a ser ocupado entre 1977 y 1979. Considerado "zona crítica", de acuerdo con el perfil socioeconómico, los habitantes de estos barrios están estigmatizados en el resto de la ciudad (CAMPOS et al., 2003). Además, tales barrios tienen entre otras características, una ocupación irregular tanto en lo que se refiere a las dimensiones y ocupación de los terrenos, como en el carácter de ilegalidad de sus nuevas construcciones, que se ubican cada vez más próximas a una gran área de riesgo y de preservación ambiental, donde existen tres manatiales de agua conviviendo con las aguas residuales de la casas de los barrios adyacentes. En estos dos barrios, es posible observar también la falta de servicios urbanos esenciales, principalmente en el área de salud, ocio y deportes. 


\section{Territorio Rural}

El asentamiento Pirituba II, con aproximadamente 300 familias agricultoras asentadas desde otras localidades, está localizado en el municipio de Itapeva, en el estado de São Paulo, en una región que presenta bajos índices de desarrollo humano (IDH 0,745, posición 558 en el ranking). Se eligió este asentamiento porque, a pesar de presentar una concentración de riquezas naturales, con florestas nativas y plantadas que comprenden un área de 600.000 hectáreas, se caracteriza como una de las regiones con los más bajos indicadores sociales del Estado de São Paulo.

\section{CONDICIONES PARA EL DESARROLLO DE LAS ACTIVIDADES DE LA INCOOP EN EL ÁMBITO DE LA UFSCAR}

La Universidad Federal de São Carlos ha realizado, en las últimas dos décadas, esfuerzos diversos para poner en práctica sus compromisos de articulación de enseñanza, investigación y extensión y para afrontar problemas sociales de diferentes naturalezas y circunstancias. Esto se viene haciendo, tanto por medio de promoción de debates conceptuales sobre la naturaleza de estas actividades y el papel de la extensión en el ámbito de la universidad, tanto interna como externa, como por la creación de instancias, instrumentos y recursos para el fortalecimiento de múltiples acciones de transformación del conocimiento sistematizado en conductas humanas. En el ámbito de las definiciones institucionales, una resolución de 1999 (UFSCar, 1999) afirma el compromiso con la indisociabilidad de las actividades de enseñanza, investigación y extensión, reiterando las actividades de extensión universitaria como parte o etapa de los procesos de producción del conocimiento y no como algo separado de estos procesos, por medio del cual la Universidad amplía el acceso al conocimiento, capacitando personas sobre el uso del conocimiento disponible. En esta normativa, se indican y definenun conjunto de actividades de extensión como formas para alcanzar el objetivo de transformar el conocimiento, que es dominio de la universidad, en accesible para la sociedad, ya sea por su propia producción o por la sistematización del conocimiento universal disponible. 
En términos de condiciones institucionales para el desarrollo de actividades de extensión tal como definidas, fueron creados, como mecanismos internos para viabilizar las actividades de extensión: núcleos articuladores de extensión; fondo de ecualización, que permite destinar recursos para apoyo de programas y proyectos de extensión; implementación de una sistemática que promueva el desarrollo de programas (como actividades duraderas y estables) y proyectos de extensión; estimulo y creación de condiciones favorables para captación de recursos y establecimiento de trabajo conjunto entre entidades capaces de promover y ampliar el compromiso social de la universidad y de su comunidad con el entorno y con las necesidades sociales que se presentan en un tiempo de complejidades crecientes; así como un Plan de Desarrollo Institucional (PDI/UFSCar), que incluye la posibilidad de creación de instancias genéricamente denominadas: Unidades Especiales de Enseñanza, Investigación y Extensión (supradepartamental, multidisciplinar y multiprofesional).

En relación con la INCOOP, la Universidad Federal de São Carlos creo y mantiene también condiciones institucionales específicas para su surgimiento y manutención. Inicialmente, con la obtención de recursos para contratación de técnicos de nivel superior, como apoyo al equipo de docentes que asumieron las actividades iniciales de la Incubadora; de modo permanente, con un espacio físico cedido por la UFSCar para el desarrollar actividades por parte de los diferentes equipos de trabajo, además del material de consumo y manutención del espacio (agua, luz y teléfono) Existe también otro espacio multiuso el cual fue una ampliación cercana al acceso y cuya función es apoyar a los núcleos y a las actividades de extensión.Actualmente, se trabaja en la perspectiva de que la Incubadora se convierta en una Unidad Especial de Enseñanza, Investigación y Extensión, con la denominación de Núcleo Multidisciplinar Integrado de Enseñanza, Investigación y Extensión en Economía Solidaria, con la condición de unidad presupuestaria, lo que deberá garantizar niveles de estabilidad institucional que puede atenuar problemas crónicos de inexistencia del cuadro técnico permanente en el ámbito administrativo y de los procesos de atendimiento a la comunidad con la participación de técnicos de nivel superior en el equipo de trabajo. 


\section{CONSIDERACIONES FINALES}

El examen continuo del método de incubación, tanto a partir de la sistematización del trabajo realizado junto a los grupos atendidos, como de las formulaciones por personas que actúan en el equipo o que participan de actividades de enseñanza desarrolladas en la Incubadora, el perfeccionamiento de su descripción - y sus efectos para la mejora de la intervención - en términos de la identificación de comportamientos más específicos que pueden ser relevantes en el proceso de atendimiento a la comunidad, de propiedades de los comportamientos más generales y específicos, así como de necesidades de producción de conocimiento sobre procedimientos y materiales para la actuación práctica del equipo de trabajo, a las cuales este mismo grupo busca responder, sea por medio de producción de conocimiento nuevo, o por medio de la transformación del conocimiento disponible en tecnología y en recursos para que el equipo y la población puedan lidiar con desafíos de grados crecientes de complejidad en el proceso de implementación de la Economía Solidaria en un contexto muy poco amistoso.

De la misma manera, las perspectivas metodológicas de actuación de la INCOOP a partir de definiciones de estrategias de desarrollo territorial y de la constitución de cadenas productivas, realizadas a partir de 2007 , vienen estableciendo nuevas demandas en cuanto a los avances conceptuales y prácticos, que inciden también en el Método de Incubación, considerando particularmente el protagonismo que emprendimientos ya incubados por la INCOOP asumieron en esta nueva etapa del funcionamiento de la Incubadora.

Finalmente, es importante resaltar que la actividad de la INCOOP en el ámbito de formación de profesionales de enseñanza superior, con la oferta semestral de la disciplina destinada a alumnos de cualquier especialidad interesados en la temática de la Economía Solidaria, ha posibilitado la permanencia de alumnos que, habiendo pasado por ella, son incluidos en diversas actividades de la INCOOP, en la condición de monitores de la disciplina, becarios en las modalidades institucionales o con recursos de proyectos gestionados por la Incubadora y aprobados por agencias de financiación de investigación, innovación tecnológica o extensión. La experiencia que resulta de este proceso apunta hacia un modo dinámico de producción de competencias, para lidiar con necesidades sociales y con 
el conocimiento sistematizado, como parte del repertorio de profesionales de nivel superior, que parece relevante compartir con otras instancias académicas.

\section{BIBLIOGRAFÍA}

ARROYO, J. C. T. (2006) Economia popular e solidária: a alavanca para o desenvolvimento sustentável e solidário. São Paulo: ed. Fundação Perseu Abramo.

CAMPOS, S. E. A. et al. (2003). O lazer cotidiano do Jardim Gonzaga - São Carlos. In: XV Encontro Nacional de Recreação e Lazer - Lazer e Trabalho: Novos Significados na Sociedade Contemporânea, Santo André (BR).

CORTEGOSO, A. L. et al. (2008). Comportamentos ao incubar empreendimentos solidários: a descrição do fazer coletivo como referencial para o fazer de cada um. En: CORTEGOSO, A. L. e LUCAS, M. (Org) Psicologia e economia solidária - Interfaces e perspectivas. São Paulo: Casa do Psicólogo, pp. 117-136.

CORTEGOSO, A. L. et al. (2005). Método de incubação como referencial para atendimento a empreendimentos solidários: a experiência da Incubadora Regional de Cooperativas Populares da UFSCar. II Jornadas Universitárias sobre Cooperativismo, Economia Solidária y Procesos Asociativos. Montevideo.

CRUZ, F. et al. (2006) "Algunas aportaciones de la Psicología Social a las Mujeres Emprendedoras en Territorios Rurales”. En: MARTÍNEZ GARCÍA, B. y LEOPOLD, L., Aportes para la Construcción de lo Colectivo, pp. 333-347. Montevideo: Psicolibros.

FÓRUM BRASILEIRO DE ECONOMIA SOLIDÁRIA (2006). Publicação de Apresentação. Brasilia : FBES.

GAIGER, L. I. (org.) (2004). Sentidos e experiências da economia solidária no Brasil. Porto Alegre: Editora da UFRGS.

ITCP-USP (2010). Incubadora de Cooperativas populares da Universidade São Paulo 2010 , disponible en http://www.itcp.usp.br/drupal/node/440. Aceso en 09 de junio 2010.

KLIKSBERG, B. (2002) América Latina: uma região de risco - pobreza, desigualdade e institucionalidade Social. Trad. de Norma Guimarães Azeredo. Brasilia: UNESCO.

MINISTERIO DO TRABALHO E EMPREGO (MTE) do Governo brasileiro, disponible en http://www.mte.gov.br/ecosolidaria/prog_incubadoras_proninc.asp. Aceso en 08 de junio 2010. 
SINGER, P. (2002a). Introdução à Economia Solidária. São Paulo: Editora Fundação Perseu Abramo.

SINGER, P (2002b). Políticas de apoio à economia solidária. Revista Sebrae, $\mathrm{n}^{\circ}$ 07, nov$\operatorname{dez} / 2002$.

UNITRABALHO 2010, disponible en http://www.unitrabalho.org.br/. Aceso en 09 de junio 2010.

UNIVERSIDADE FEDERAL DE SÃO CARLOS (1999). Portaria GR 664/99. Disponible en http://www2.ufscar.br/interface_frames/index.php?link=http://www.proex.ufscar.br 